\title{
Primary progressive multiple sclerosis overlapping with anti-GAD and anti-Hu antibodies positive neurological syndromes
}

\author{
Pavel Štouračl ${ }^{10}$, Jana Bednářová ${ }^{2}$, Zbyšek Pavelek ${ }^{3}$, Martin Vališ ${ }^{3}$ \\ ${ }^{1}$ Department of Neurology, University Hospital Brno and Faculty of Medicine, Masaryk University, Brno, Czech Republic \\ ${ }^{2}$ Department of Clinical Microbiology, University Hospital Brno and Faculty of Medicine, Masaryk University, Brno, Czech Republic \\ ${ }^{3}$ Department of Neurology, University Hospital Hradec Králové and Faculty of Medicine, Charles University, Hradec Králové, Czech Republic
}

Key words: anti-Hu, anti-GAD antibodies, primary progressive multiple sclerosis, overlapping syndromes

(Neurol Neurochir Pol 2022; 56 (2): 187-190)

\section{To the Editors}

Although still rare, there have been reports describing autoimmune demyelinating disorders associated with specific antibodies which overlap with multiple sclerosis (MS) and other demyelinating syndromes in clinical, radiological and immunological features.

A relationship between anti-myelin-associated glycoprotein (MOG) syndrome, anti-aquaporin-4 (AQ-4) neuromyelitis optica spectrum disorders, and anti-N-methyl-D-aspartate receptor (NMDAR) encephalitis has been described. The opportunity of discovering yet unknown associations is based on the presumption of the co-occurrence of immune mediated diseases [1]. The association of multiple sclerosis with glutamic acid decarboxylase (GAD) neurological syndromes has only been reported in one case $[2,3]$. The associated neurological syndromes include stiff-person syndrome (SPS), cerebellar ataxia, epilepsy, and limbic encephalitis. Paraneoplastic origin is rare, and comorbidities include autoimmune thyreoiditis, pernicious anaemia and vitiligo [4].

More than $85 \%$ of patients with anti-Hu antibodies harbour small cell lung cancer (SCLC) or, less frequently, other tumours. Anti-Hu antibodies can be found in the sera of $2 \%$ of patients without a tumour after five years, but these antibodies are not detected in normal subjects or in other neurological disorders [5].

We present one case of non-paraneoplastic anti- $\mathrm{Hu}$ brainstem syndrome and one case of progressive $\mathrm{GAD}$ ataxia syndrome associated with primary progressive multiple sclerosis (PP-MS).

\section{Case 1}

A 57-year-old woman had been treated for autoimmune thyreoiditis with levothyroxinum natricum (Euthyrox tablets) since December 2018. No cardiovascular or rheumatoid diseases were present, including negative laboratory screeening. In 2014, she suffered from vertigo, and mild balance and coordination difficulties. In 2018, her condition worsened with additionally reported diplopia, dysarthria, unsteady walking with falls, fatigue and paraesthesias. Neurological examination discovered limb and gait ataxia, supranuclear vertical gaze palsy, diplopia, low reflexes and imperative micturion with EDSS grade 5.0. Tests for hereditary ataxias and anti-MOG and anti-AQ4 were negative. Anti-GAD antibodies were detected in serum. Paraneoplastic origin of anti-GAD antibodies was excluded by negative positron emission tomography (PET) of the whole body. MRI brain scan discovered multiple hyperintense lesions predominantly in juxtacortical and periventricular locations, fulfilling the $2017 \mathrm{McD}$ onald criteria for multiple sclerosis. No lesions or atrophy were found in the cerebellum. Cerebrospinal fluid analysis (CSF) showed normal cell count and protein content, but positive oligoclonal IgG bands in CSF and serum (n-5/n-1). MRZ (measles, rubella, zoster) reaction was positive. The treatment included intravenous methylprednisolone, intravenous immunoglobulins, and plasmapheresis,

Address for correspondence: Pavel Stourac, University Hospital Brno, Jihlavska 20, 62500 Brno, Czech Republic; e-mail: Stourac.pavel@fnbrno.cz Received: 20.05.2021 Accepted: 29.09.2021 Early publication date: 27.10.2021

This article is available in open access under Creative Common Attribution-Non-Commercial-No Derivatives 4.0 International (CC BY-NC-ND 4.0) license, allowing to download articles and share them with others as long as they credit the authors and the publisher, but without permission to change them in any way or use them commercially. 
with temporary stabilisation mostly after the plasmapheresis. The patient's walking worsened, with the need of the support of a walking stick (EDSS 6.0) in 2020. MRI examinations in 2020, 2021 confirmed multiple white matter hyperintense lesions, some of them with T1-hypointense correlates and no lesions in the cerebellum. CSF analysis showed an increased number of oligoclonal IgG bands in CSF and serum (n-11/n-6). Increased kappa free light chain ( $\kappa$ FLC) index value of 12.3 (normal range 0.8-5.9) was detected. Anti-GAD antibodies persisted in serum and cerebrospinal fluid. These CSF results are consistent with the so-called disease-related pattern for multiple sclerosis. Rituximab (600 mg intravenously) administered every six months led to clinical stabilisation. The patient fulfilled the diagnostic criteria for PP-MS (2017) by confirmed EDSS progression, hyperintense lesions in typical locations in MRI brain scan, and by the presence of oligoclonal $\operatorname{IgG}$ bands in CSF. PP-MS, GAD-associated cerebellar ataxia, and autoimmune thyreoiditis represent a unique trio of immune-mediated disorders with overlapping symptoms.

\section{Case 2}

A 69-year-old man without cardiovascular, rheumatoid or other significant comorbidities had developed brain stem syndrome with nystagmus, diplopia, vertigo, dysphagia, dysarthria, gait and limb ataxia in 1998. Initial MRI brain scan was normal. Anti-Hu antibodies were detected in serum and CSF, with otherwise normal CSF findings. Malignancy was excluded by repeated PET and bronchoscopy. MRI brain scan in 2006 revealed mild cerebellar atrophy but no lesions. The patient's condition insidiously worsened and in 2012 he started to use a walking stick, and intermittently a wheelchair (EDSS 6.5). MRI brain scans during the period 2014-2020 showed new hyperintense lesions in juxtacortical, periventricular and brainstem locations, fulfilling MRI criteria for multiple sclerosis. Anti-Hu antibodies were constantly positive in serum and CSF. CSF analysis showed mild pleocytosis, intrathecal IgG synthesis confirmed by oligoclonal IgG bands in CSF and serum (n-8/n-3) in 2021.

The patient fulfills diagnostic criteria for PP-MS with severe progressive cerebellar syndrome, walking impairment reflected by EDSS, hyperintense lesions in typical locations in MRI brain scan, positive oligoclonal IgG bands in CSF in parallel with anti-Hu brainstem syndrome without tumour. Treatment with intravenous methylprednisolone and symptomatic treatment of fatigue and spasticity were partly effective.

This case has two unique aspects: firstly displaying clinically associated and overlapping PP-MS and anti-Hu brainstem syndrome; and secondly a very unusual long-term anti-Hu positivity of non-paraneoplastic origin.

There is evidence of a different immunological mechanism in both disorders. The pathological role of anti-GAD and anti-Hu antibodies is still a matter of debate. GAD-related neurological syndromes are uncommon and account for about

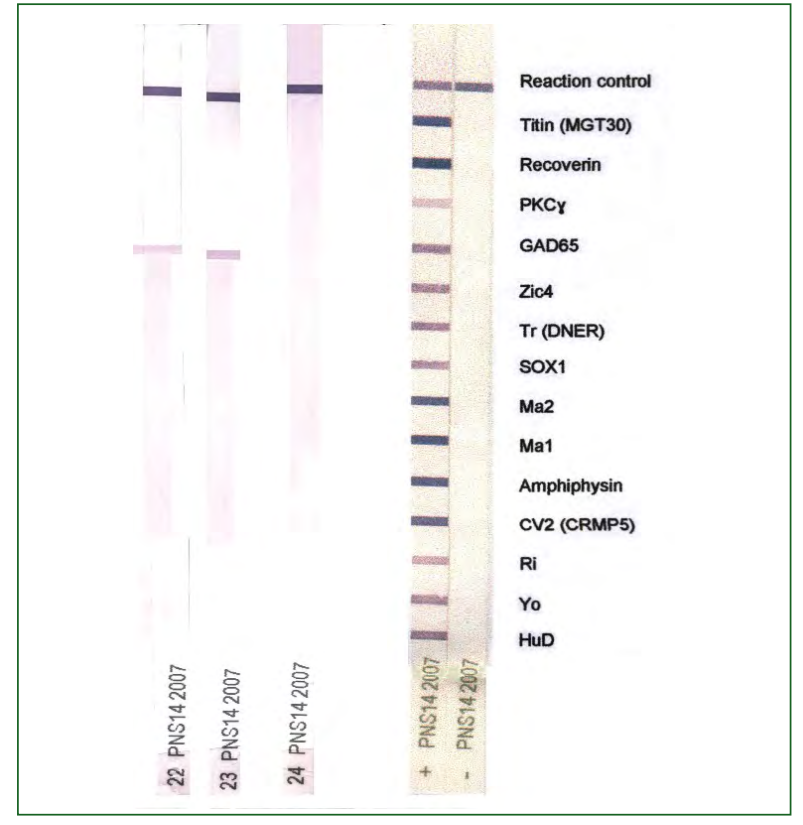

Figure 1. Immunoblot with positive anti-GAD65 antibodies in serum and cerebrospinal fluid (left to right) of patient with GAD cerebellar ataxia syndrome (Ravo, Germany)

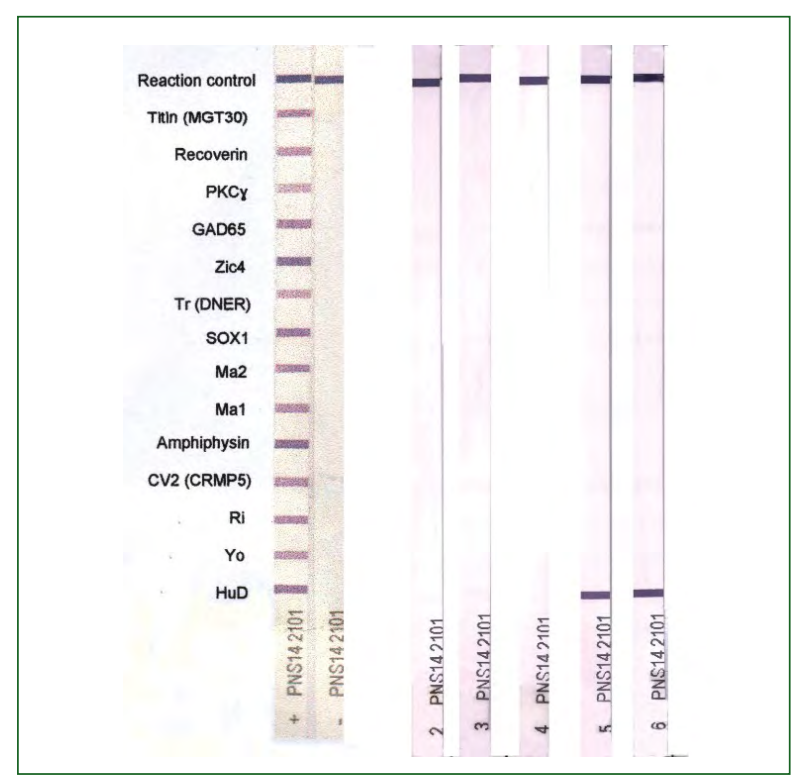

Figure 2. Immunoblot with positive onconeural anti-Hu antibodies in serum and cerebrospinal fluid (left to right) of patient with anti-Hu positive brainstem syndrome of non-paraneoplastic origin (Ravo,Germany)

$2 \%$ of sporadic progressive cerebellar ataxia and for $12 \%$ of cerebellar ataxia of unknown origin [6, 7]. Anti-GAD antibodies occur in SPS syndrome, progressive encephalomyelitis with rigidity and myoclonus (PERM), cerebellar ataxia, limbic and extralimbic encephalitis, epilepsy and oculomotor dysfunction and nystagmus [8]. Increased titre of anti-GAD antibodies is 


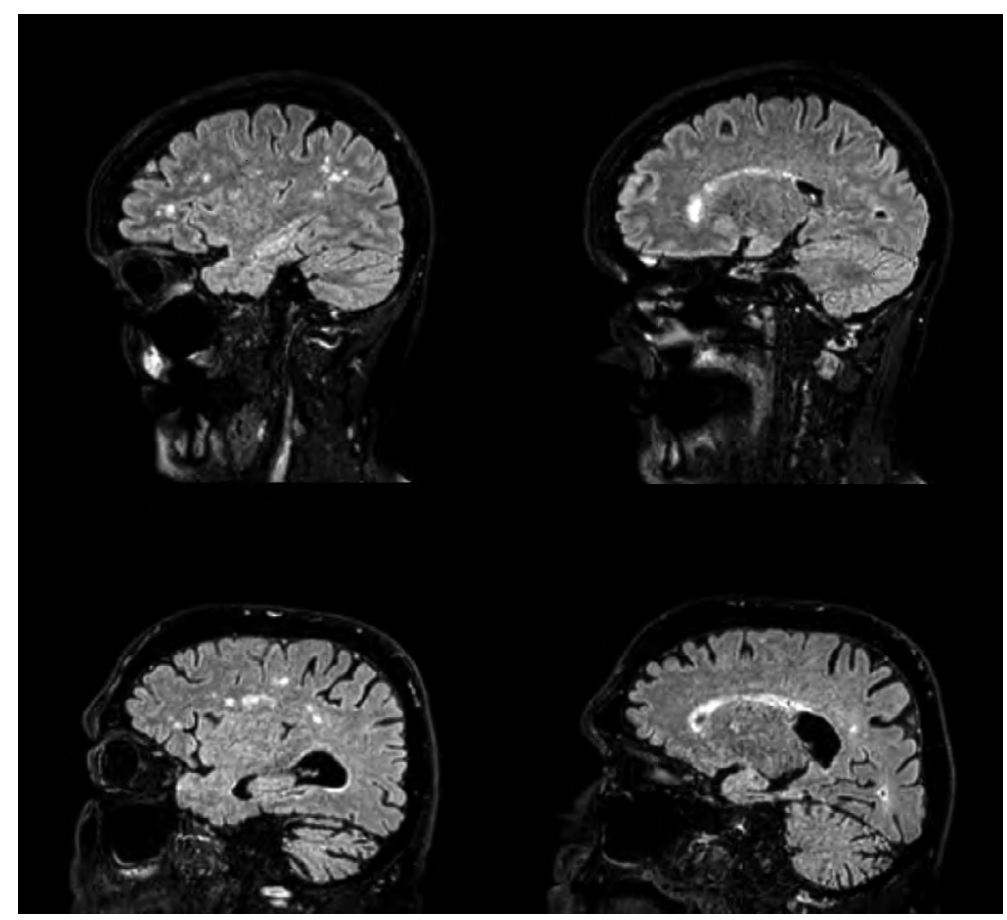

Figure 3. MRI of brain demonstrating typical finding of MS in 3D fluid-attenuated inversion recover (FLAIR) images of brain in sagittal plane. Scans a and b belong to Case 1 ; scans $\mathrm{c}$ and $\mathrm{d}$ belong to Case 2

neither a non-specific epiphenomenon of neuronal damage, nor a common feature of recognised neuroimmunological disorders. Anti-GAD antibodies may be a pathogenetic agent or a marker for an ongoing autoimmune process, or both [9]. This patient's paraesthesias, fatigue, cognitive disorder and imperative micturion belong to a typical profile of PP-MS. The patient also has autoimmune thyreoiditis which is associated with SPS/cerebellar ataxia syndrome in c.50\% of cases [10].

In GAD-associated cerebellar ataxia, MRI usually shows cerebellar atrophy with preservation of medulla oblongata [11]. The patient's MRI brain scan showed multiple hyperintense lesions of typical locations up to $12 \mathrm{~mm}$ in size fulfilling MS diagnostic criteria. There is only one report of a patient developing epilepsy with extralimbic encephalitis with cortico-subcortical lesions in T2W/FLAIR MRI [12]. CSF analysis showed oligoclonal IgG bands in CSF and elevated kappa free light chains index with a high sensitivity (89-95\%) and specificity (95-100\%) for multiple sclerosis [13]. A positive MRZ reaction supported the MS diagnosis [14].

In the diffential diagnosis, we excluded compressive lesions, systemic autoimmune disorders, sarcoidosis, CNS infections including HTLV-1, syphilis, borreliosis and inherited disorders. The paraneoplastic syndromes with onconeural anti-Hu antibodies present disorders as sensory neuronopathy/ encephalomyelitis, limbic encephalitis, brainstem encephalopathy, opsoclonus-myoclonus and myelopathy. Anti-Hu antibodies have $99 \%$ specificity and $82 \%$ sensitivity in detecting paraneoplastic neurological syndromes [15]. There is a reported prevalence of $32 \%$ for brainstem dysfunction and $25 \%$ for cerebellar dysfunction in anti-Hu positive patients.

The absence of a tumour and the permanent positivity of anti-Hu antibodies for 23 years, in spite of repeated oncological screeening including PET investigation, are unique features of this case. The continuous deterioration in walking paralled by an increasing number of hyperintense lesions in typical brain locations and positive CSF findings confirmed the diagnosis of PP-MS associated with anti-Hu brainstem syndrome. Compressive, autoimmune, infectious and inherited disorders were excluded in the differential diagnosis. There are no reports of long-term non-paraneoplastic anti-Hu syndromes, but there is a hypothesis that immune reaction could eliminate a tumour localised in situ at the beginning of oncogenesis, although this has not been proved experimentally yet [16].

Both these cases are important in terms of differential diagnoses in respect of different immune mechanisms, treatment and prognosis.

Conflict of interest: None.

Funding: This work was supported by the Ministry of Health of the Czech Republic, ref.-RVO (FNBr,65269705).

\section{References}

1. Titulaer MJ, Höftberger R, lizuka T, et al. Overlapping demyelinating syndromes and anti-N-methyl-D-aspartate receptor encephalitis. Ann Neurol. 2014; 75(3): 411-428, doi: 10.1002/ana.24117, indexed in Pubmed: 24700511. 
2. Naik S, Kolikonda MK, Lippmann S. Progressive encephalomyelitis with rigidity: stiff person syndrome variant associated with multiple sclerosis. Prim Care Companion CNS Disord. 2019; 21(1), doi: 10.4088/PCC.18I02360, indexed in Pubmed: 30762977.

3. Thompson A, Banwell B, Barkhof F, et al. Diagnosis of multiple sclerosis: 2017 revisions of the McDonald criteria. The Lancet Neurology. 2018; 17(2): 162-173, doi: 10.1016/s1474-4422(17)30470-2.

4. Graus F, Saiz A, Dalmau J. GAD antibodies in neurological disorders insights and challenges. Nat Rev Neurol. 2020; 16(7): 353-365, doi: 10.1038/s41582-020-0359-x, indexed in Pubmed: 32457440.

5. Pellizza F, Nobile-Orazio O, Giometto B. Neuronal Nuclear Antibodies, Type 1(Hu). In: Shoenfeld Y, Meroni Pl, Gershwin ME. ed. Autoantibodies. Elsevier 2014.

6. Hadjivassiliou M, Martindale J, Shanmugarajah P, et al. Causes of progressive cerebellar ataxia: prospective evaluation of 1500 patients. J Neurol Neurosurg Psychiatry. 2017; 88(4): 301-309, doi: 10.1136/ jnnp-2016-314863, indexed in Pubmed: 27965395.

7. Nanri K, Okuma M, Sato S, et al. Prevalence of autoantibodies and the efficacy of immunotherapy for autoimmune cerebellar ataxia. Intern Med. 2016; 55(5): 449-454, doi: 10.2169/internalmedicine.55.5156, indexed in Pubmed: 26935362.

8. Herard K, Khanni J, Hibbert KA, et al. Neurological disorders associated with glutamic acid decarboxylase antibodies. Cureus. 2019, doi: 10.7759/cureus. 4738.

9. Meinck HM, Faber L, Morgenthaler N, et al. Antibodies against glutamic acid decarboxylase: prevalence in neurological diseases. J Neurol Neurosurg Psychiatry. 2001; 71(1): 100-103, doi: 10.1136/ jnnp.71.1.100, indexed in Pubmed: 11413272.
10. Dalakas MC, Fujii M, Li M, et al. The clinical spectrum of anti-GAD antibody-positive patients with stiff-person syndrome. Neurology. 2000; 55(10): 1531-1535, doi: 10.1212/wnl.55.10.1531, indexed in Pubmed: 11094109.

11. Honnorat J, Saiz A, Giometto B, et al. Cerebellar ataxia with anti-glutamic acid decarboxylase antibodies: study of 14 patients. Arch Neurol. 2001; 58(2): 225-230, doi: 10.1001/archneur.58.2.225, indexed in Pubmed: 11176960.

12. Cianci V, Labate A, et al. Non-paraneoplastic limbic encephalitis characterized by mesio-temporal seizures and extratemporal lesions: case report . Seizure. 2010; 19(7): 446-449, doi: 10.1016/j.seizure.2010.06.002, indexed in Pubmed: 20598587.

13. Duell F, Evertsson B, Al Nimer F, et al. Diagnostic accuracy of intrathecal kappa free light chains compared with OCBs in MS. Neurol Neuroimmunol Neuroinflamm. 2020; 7(4), doi: 10.1212/ NXI.0000000000000775, indexed in Pubmed: 32527760.

14. Hottenrott T, Dersch R, Berger B, et al. The MRZ reaction in primary progressive multiple sclerosis. Fluids Barriers CNS. 2017; 14(1): 2, doi: 10.1186/s12987-016-0049-7, indexed in Pubmed: 28166789.

15. Senties-Madrid H, Vega-Boada F. Paraneoplastic syndromes associated with anti-Hu antibodies. Isr Med Assoc J. 2001; 3(2): 94-103, indexed in Pubmed: 11344832.

16. Li J, Lin W. Various clinical features of patients with anti-Hu associated paraneoplastic neurological syndromes: An observational study. Medicine (Baltimore). 2018; 97(18): e0649, doi: 10.1097/MD.0000000000010649, indexed in Pubmed: 29718880. 